INDUSTRIE

PAR PHILIPPE LORINO, JACQUES NEFUSSI

\title{
Tertiarisation des fflères et reconstruction du Sens à travers des réCits collectifs
}

Avec la « tertiarisation », les entreprises doivent produire, non plus des produits, mais des « solutions » intégrant la valeur d'usage du client. Cette mutation renouvelle en profondeur les logiques économiques et organisationnelles à l'œuvre dans les entreprises industrielles et agricoles. Elle exige une transformation radicale du cadre d'interprétation de l'activité pour les différents acteurs de la chaîne de valeur: fournisseurs, salariés, clients. Dans cet article, les auteurs s'intéressent aux enjeux managériaux liés à cette transformation et donnent une lecture théorique. Les acteurs de la chaîne de valeur concernés se trouvent confrontés à la remise en cause des cadres de compréhension de leur activité et à la mise en échec des récits par lesquels ils avaient I'habitude de faire sens de leur coopération. Ils doivent donc construire une nouvelle intelligibilité de l'activité collective.

\section{I. - LA TERTIARISATION DE L'AGRICULTURE ET DE L'INDUSTRIE}

\section{Produits ou « solutions »?}

Les activités économiques (industrielles, agricoles, de services) connaissent une mutation profonde que l'on désigne communément par le terme de «tertiarisation » (Gadrey, Delaunay, 1987 ; De Bandt, 1991 ; De Bandt, Gadrey, 1994). Avec la «tertiarisation » se transforment et se complexifient les modes de création de valeur, par une reconstruction plus ou moins continue des usages: les questions «quel besoin? », « quelle solution? » sont reposées de façon récurrente, alors que l'industrie fordienne établissait une définition stable et standardisée du produit et de son usage (Levitt, 1981).

La démarche «solution » se substitue alors à la démarche «produit », tant dans les services de masse traditionnels que dans l'industrie manufacturière. Une solution est un ensemble de modes relationnels avec le client et d'outils qui fonde une valeur d'usage. Xerox cesse de se définir comme producteur de copieurs pour devenir « la compagnie des solutions de gestion documentaire » («the document company ») (Allaire, 1992). 
Pour construire des solutions de transport « porte à porte » intégrant les divers modes de transport et visant, selon les cas, les migrations quotidiennes domicile-travail, les voyages d'affaires ou les déplacements de loisirs, la SNCF passe des accords avec les régies de transports urbains des villes ou avec les aéroports et Air France. Objectifs : coordonner les horaires, mettre en cohérence les localisations spatiales des gares, des aéroports et des arrêts d'autobus, développer des formules tarifaires multimodales. La vente de kilomètres-passagers n'est plus l'alpha et l'oméga du transport ferroviaire.

À travers cette mutation se joue en fait l'intégration active du client dans la conception et la production des éléments de valeur. La création de valeur se déplace de la production de produits pré-spécifiés permettant de répondre à des besoins standardisés, plus ou moins stables et prédéfinis par le fournisseur, vers la construction de problèmes toujours renouvelés, la conception et la mise en œuvre de solutions où le client est omniprésent. Dans la production de biens sont alors remises en cause les bases tayloriennes et fordiennes de l'organisation productive: de moins en moins de répétitivité et de standardisation au profit d'une plus grande différenciation. La séparation entre conception et exécution, au cœur du taylorisme, est remise en cause. Dans une activité de service, la solution est, au moins en partie, conçue, produite, et livrée d'un seul et même mouvement. Elle est même coconçue, coproduite et comise en œuvre par le fournisseur de service et le client, de manière itérative.

Les allers-retours récurrents d'une phase à l'autre de la chaîne de valeur en rendent difficile l'organisation séquentielle cloisonnée par phases. Or le modèle « industriel » classique supposait qu'on pouvait introduire une césure forte et stable dans les processus coopératifs, entre un amont et un aval, par exemple entre conception et production, ou entre conception - production et utilisation. Ces césures correspondaient souvent à l'irruption du marché dans la chaîne. L'amont vendait, l'aval achetait, les cloisonnements entre phases de la chaîne de valeur s'identifiant alors à l'existence de marchés de produits intermédiaires: le marché du pneumatique ou des systèmes de climatisation dans la chaîne de valeur automobile, le marché du transport dans la chaîne de valeur logistique. Avec la tertiarisation, la définition de ces marchés devient plus problématique. Lorsque Xerox propose une solution intégrée de gestion documentaire à un client professionnel, la place du marché des copieurs est assez difficile à délimiter.

Cependant, ce processus rencontre des limites avec l'effort de rationalisation dans certaines activités du secteur tertiaire telles que les centres d'appel, où le modèle industriel taylorisé est introduit pour bénéficier d'économies d'échelle. La notion de tertiarisation à laquelle nous recourons ici doit donc être vue comme un type précis de transformation stratégique et organisationnelle, qui ne coincide pas avec la notion macroéconomique de service. La tertiarisation, fondée sur des réponses spécifiques à des besoins de clients plus ou moins fortement différenciés, est une réponse à des niveaux de complexité et d'incertitude élevés. Certains secteurs de services, ou certaines fonctions au sein d'une activité de service, s'industrialisent au sens taylorien au moment même où certains secteurs manufacturiers, ou certaines fonctions au sein d'une industrie manufacturière, se ter- 
tiarisent. Des modèles intermédiaires, par exemple la personnalisation de masse, émergent. Tertiarisation et taylorisation se développent simultanément. Les chaînes de valeur se re-fractionnent de manière distincte. De nouveaux marchés émergent, là où prédominaient des opérations internes aux entreprises. Des domaines de services personnalisés se standardisent et s'industrialisent. Le logiciel informatique est emblématique de ces transformations : dans les années 1970 et 1980 , la plupart des utilisateurs industriels de l'informatique disposaient de solutions « maison » spécifiques. La montée en puissance des performances des progiciels (logiciels standard de comptabilité, de paie, de gestion logistique, etc.) à des coûts inaccessibles aux logiciels spécifiques a eu raison de cette situation. Rares sont aujourd'hui les entreprises qui disposent encore de solutions « maison » dans ces grands domaines applicatifs. Mais en s'éloignant du matériel et du logiciel informatiques, la valeur ajoutée spécifique s'est déplacée vers le développement d'usages managériaux de plus en plus élaborés et complexes.

\section{Les actions collectives conjointes au cour de la tertiarisation}

La tertiarisation remet l'action collective au premier plan de l'étude des organisations. Nous définirons l'organisation comme système d'action socialement articulé par la division du travail et la mutualisation de ressources. Elle ne peut opérer que par la capacité de coopérer des acteurs, ce qui exige notamment un seuil minimum d'intelligibilité mutuelle. Les processus combinés de conception, production et livraison de solutions répondent au modèle de l'action collective conjointe, par opposition à l'action collective commune (Vernant). L'action collective organisée peut en effet revêtir deux figures essentielles: l'action commune (les acteurs sont appelés à exécuter la même activité ou des activités similaires, par exemple le même métier: c'est alors la similitude qui est mise en avant, comme c'est le cas dans une communauté de pratique) ou l'action conjointe (les acteurs sont appelés à faire des choses différentes : vendre, produire, facturer, mais de manière coordonnée, selon un schéma de division du travail; c'est alors la complémentarité qui est mise en avant). La coopération, au moins bipolaire (client/fournisseur) et souvent multipolaire (plusieurs fournisseurs, parfois même plusieurs clients), dans la chaîne de valeur du service met évidemment en jeu des activités et des compétences complémentaires et non identiques, selon le schéma de l'action conjointe plutôt que celui de l'action commune.

Le service est globalement une transaction transformatrice du monde (par exemple, le transport quotidien de voyageurs se rendant à leur travail ou le financement de projets immobiliers changent le « paysage » social dans lequel nous vivons). Cette transaction (« trans-action », action collective « traversant » les divers acteurs qui y sont engagés pour s'exercer sur le monde) repose sur des interactions ( inter-actions », actions que les acteurs exercent les uns sur les autres) entre les acteurs qui coopèrent pour la rendre possible, notamment les utilisateurs finaux. Lorsque la transaction, l'action conjointe, répond à un modèle répétitif et stable, les interactions entre les acteurs peuvent être normées. « De temps en temps », on conçoit les modes de coordination (souvent en même temps que le produit final), et 
entre ces efforts périodiques de conception, on applique des normes. Avec la tertiarisation la part du récurrent diminue et la part du spécifique augmente, ce qui implique la nécessité de reconstruire continûment les modes de coordination. Les interactions entre acteurs ne peuvent plus simplement se conformer à un modèle préétabli : elles doivent satisfaire à des exigences beaucoup plus élevées de communication permanente dans une reconception au cas par cas du service à rendre et des coordinations nécessaires. La tertiarisation repose donc sur une coopérativité renforcée: chacun des acteurs de la chaîne possède une part de la compétence collective requise pour répondre aux besoins du client (Gadrey, Zarifian).

\section{Maintien de la coopérativité par la réflexivité dans la production de solutions}

Dans un contexte de tertiarisation, où les normes de coordination se redéfinissent partiellement en situation, la coopérativité ne peut se maintenir à travers le temps que par la réflexivité, déjà mise en évidence, par exemple, dans les travaux de l'école de Palo Alto ou ceux d'Argyris et Schön (1978) avec la notion de «deutero-learning »: les acteurs de la chaîne du service doivent faire retour sur leur action conjointe et ses conditions, y compris ses outils, pour se comprendre. Si la coopérativité s'appuie ainsi sur la réflexivité, réciproquement, la réflexivité du groupe sur le processus d'action conjointe est coopérative: pour faire retour sur la prestation de service et en saisir la cohérence d'ensemble, il faut que les acteurs de la chaîne coopérent, car aucun n'est surplombant, leurs compétences se complètent. La combinaison de la réflexivité et de la coopérativité des agents est essentielle dans le cadre du processus de tertiarisation. « Parlant à, et avec, autrui, le locuteur se parle à lui-même », constate Denis Vernant (1997). Grâce à cette « conversation » avec autrui, l'acteur peut mettre sa propre activité à distance pour en faire un objet sur lequel agir de manière réflexive. Le regard de l'autre « objective » son activité. Mais cette réflexivité s'exerce d'emblée dans un contexte social. Inversant l'expression de Denis Vernant, nous pourrions dire aussi : «se parlant à lui-même, le locuteur parle avec autrui ». Lorsqu'il se penche sur les outils et les conditions de son activité, l'agent se trouve immédiatement pris dans l'organisation d'une activité collective (division du travail, partage de ressources, partage d'outils, délégations de pouvoir, etc.).

\section{La construction du sens dans l'action collective conjointe nécessite une enquête collective}

L'action conjointe est marquée d'une double séparation: d'une part, la séparation des acteurs entre eux, car la division technique du travail leur impartit des activités différentes, porteuses de connaissances différentes; d'autre part, la séparation entre les acteurs du service et le client final: la division économique du travail (les diverses entreprises engagées dans la prestation de service sont en concurrence pour se partager la marge) limite l'accès à l'activité du client pour comprendre les problèmes qu'il rencontre. Dans cette situation de double séparation, les acteurs disposent d'une autonomie d'interprétation et coopèrent avec des schémas d'interprétation structurellement différents, car chacun possède sa propre sémantique, sa propre identité culturelle, son propre contexte profes- 
sionnel et sa propre histoire. La solution à coproduire n'est pas donnée, il n'y a pas de « vérité positive » du processus de création de valeur par le service, mais des vues diverses plus ou moins compatibles entre elles. Les acteurs engagés dans une coproduction de service doivent donc construire un système de signes, un langage, qui leur permette de communiquer, de se comprendre et de se coordonner. Le sens global de leur action conjointe est donné par le client (dont la présence fonde le sens d'une utilité et d'un résultat à produire), mais ce sens doit être relayé ensuite dans la réalité opérationnelle et les coopérations concrètes entre acteurs par un système de signes donnant accès à des significations opératoires (Vygotski, 1997). La coopération n'est rendue possible que par la capacité des acteurs à faire sens de leur interaction (Weick, 2001), en construisant de manière continue une intelligibilité mutuelle, comme dans une conversation (la conversation se construit de manière coopérative par la capacité des locuteurs à construire du sens dans leur interaction, et ce, de manière continûment renouvelée). Les acteurs du service font sens pour agir, en projetant leur action dans un espace de signification particulier, celui du service à rendre au client, par le truchement de signes. Les signes n'entretiennent pas un rapport de vérité (de reproduction fidèle) avec le monde où se meuvent les acteurs, mais un rapport de signification (Peirce, 1978) : ils permettent aux acteurs de « faire sens » des situations pour agir.

Les approches pragmatiques (actions) et sémiotiques (signes/sens) sont ainsi liées étroitement dans la lecture de la tertiarisation que nous proposons.
En effet, la production d'un langage commun sous des formes multiples (économique: coûts, marges, technique, qualiticien, sécuritaire, etc.), constitue un enjeu central du processus de tertiarisation: elle répond à une exigence pragmatique, une intention et une volonté de résultat. Elle est en permanence tirée par le client et les demandes qu'il formule pour résoudre ses propres difficultés. L'exigence du client apparaissant ainsi comme un problème à résoudre, elle impose de déclencher une enquête au sens de John Dewey (1993).

L'enquête n'est pas une explicitation du tacite, la révélation d'une vérité cachée, comme le laisserait entendre la notion d'externalisation dans le modèle de la « spirale de la connaissance » de Nonaka et Takeuchi (1995). Elle est (re)construction du jugement des acteurs à partir d'un doute initial, en l'occurrence la demande du client qui engendre des «écarts » par rapport aux habitudes acquises. L'enquête n'est pas non plus mise en œuvre désincarnée et hors le temps d'un algorithme de résolution de problème. Elle est processus d'action (au sens policier, l'enquêteur agit: il cherche des indices, construit des hypothèses, les vérifie, interroge des témoins, surveille..., et cela exige des ressources et du temps). Le processus d'enquête est coopératif (l'enquête engage plusieurs acteurs) et réflexif (les acteurs enquêtent sur leur propre processus de production de solution).

L'enquête collective sur la production du service est instrumentée par toutes sortes d'artefacts: des glossaires, des outils matériels, des règles formelles ou informelles, des récits, un diagramme de processus, un guide d'entretien, un modèle de simulation de la chaîne de valeur. Ces artefacts, par 
leur existence « objective », qu'ils soient matériels ou immatériels, sont à la fois des contraintes et des ressources pour l'action: contraintes parce qu'on ne peut pas faire n'importe quoi avec eux, ils « résistent »; ressources parce qu'ils « canalisent » l'action, évitent de tout réinventer dans chaque situation et permettent des « économies d'attention ». Toutefois ils ne déterminent pas totalement l'action et laissent une marge aux interprétations des acteurs, notamment dans la manière de les utiliser: des «styles » personnels (Clot, 1999/2002; Clot et Faïta, 2000) se développent.

\section{Le caractère instrumental de la « solution »}

Un instrument est la combinaison d'un artefact « objectif » (un modèle mathématique, un logiciel) et d'un schème d'utilisation « subjectif »(Rabardel, 1999/2002). Les instruments mobilisés dans la prestation de service ont pour fonction de créer des régularités (par exemple, assurer une convergence suffisante de la vision qu'ont les divers acteurs du déroulement de la prestation, assurer sa continuité dans le temps), mais aussi de révéler/construire des écarts (par exemple, l'écart entre ce que A pense faire et ce que B croit que A fait, la hiérarchisation différente des priorités, l'écart entre la perception du besoin final et son expression par les utilisateurs...).

Dans les solutions, les produits (le photocopieur, le logiciel de gestion documentaire, le réseau local, la carcasse de porc) sont des instruments parmi d'autres, au sens de la théorie instrumentale de l'activité (Vygotski, 1997 ; Rabardel, 1999/2002). Ils ne sont pas porteurs de sens en eux-mêmes, ni points d'aboutissement finalisés des processus de conception et de production. Le sens se déplace du produit à l'usage et les produits ne prennent sens que comme outils médiatisant un usage, supports d'une activité utilisatrice. Instruments, ils ont une double nature d'artefacts objectifs conçus et produits, comme les objets manufacturés d'hier, et de schèmes d'utilisation subjectifs, qui seuls peuvent leur conférer une quelconque valeur, la valeur étant attachée à un usage. Les schèmes d'utilisation des produits participent ainsi de la solution et de la valeur créée. Même des biens manufacturés à l'identité industrielle solide connaissent cette évolution: le jour n'est peut-être pas loin où les compagnies aériennes cesseront totalement d'acheter des avions, pour ne plus acheter que de la « capacité de transporter », sous forme de solutions intégrant la location d'avions, des services de contrôle et de maintenance, l'adaptation récurrente des aménagements intérieurs des appareils...

\section{Le rôle du récit dans l'approche pragmatique du changement organisationnel}

Le récit joue un rôle essentiel dans ce processus de changement, car il permet aux acteurs de la chaîne de valeur de donner un sens nouveau à leur articulation avec les clients. Nous emprunterons ici à Paul Ricœur (1984) sa définition du récit comme « activité mimétique ayant pour objet la mise en intrigue de l'expérience vive ». Face au caractère chaotique, « discordant » selon les termes de Ricœur, de l'expérience vive, la narration recompose les faits de manière créative pour rendre l'action intelligible. Cette reproduction mimétique n'est donc pas une copie passive, mais un réagencement des faits et du temps. Face au client, qui apparaît comme source d'éven- 
tuelles contradictions, l'enquête est un processus narratif collectif, qui vise à construire collectivement, de manière « polyphonique », un récit plausible du processus « service ». Ce récit plausible n'est pas la vérité du processus: il est un récit accepté par tous et dont on va tester la validité pratique. On se distingue, par ce statut « modeste » de l'enquête (pas de rapport de «vérité » avec le monde, mais plutôt un rapport de sens), des analyses positivistes telles que le taylorisme (existence d'une " one best way » scientifique) et de la démarche clinique de Chris Argyris et Donald Schön (1978). Ces derniers veulent contribuer à ce que les acteurs, non seulement surmontent la difficulté pratique posée initialement, mais en comprennent aussi les «causes » (au sens de la causalité positive des sciences dures: les routines défensives, par exemple) pour essayer d'y remédier. Les théories cliniques se situent dans un rapport de « vérité » ou de « santé » avec l'organisation, là où l'enquête se contente de reconstruire le jugement pour ouvrir de nouvelles possibilités d'action et (r)établir un état de signification pour les acteurs plutôt qu'un état de vérité.

L'enquête procède par combinaison de récits partiels (restituant la dimension temporelle de l'action, le « déroulement » diachronique des opérations) et de descriptions (restituant la dimension spatiale de l'action: comment s'articulent les diverses actions à un instant donné). Elle transforme et combine les récits individuels de départ, historiques, souvent parallèles et non communicants (chacun conte « son » histoire), en description (le récit de chaque acteur est progressivement intégré dans une description partagée du processus). Puis elle retransforme la description du processus en récit, mais cette fois en « récit diagnostic » (« voilà comment, collectivement, nous sommes prêts à raconter la genèse explicative de la situation présente ») et en « récit utopique » (« voilà ce que nous allons faire ensemble désormais »). De cette manière, les récits, initialement historiques et séparés, se combinent dans un récit collectif utopique, en se pliant au passage à l'impératif descriptif qui les contraint et les unit: produire une image du processus présentant un minimum de cohérence et d'opérationnalité. Le récit, pour «mettre en intrigue », peut prendre plusieurs formes: rapports, notes de synthèse, jeux de transparents, plans d'actions, etc. Comme dans tout récit, les énoncés ont des référents (Charaudeau et Maingueneau, 2002) fondés sur la perception que chaque acteur de la chaîne de valeur a de son activité dans la division du travail. Cette perception provient de son expérience immédiate et de ses schèmes d'interprétation.

La production de récit par les acteurs de la chaîne de valeur apparaît ainsi comme un instrument de conduite du changement, qui tire son efficacité de plusieurs facteurs:

- Les référents sous-jacents aux diverses opinions exprimées doivent être compris, validés et partagés par la communauté de travail à partir d'une nouvelle production de connaissances. Ce travail permet un élargissement du champ référentiel de chaque acteur (en produisant un certain niveau d'intelligibilité mutuelle des expériences particulières).

- Les schèmes d'interprétations sont souvent différents, voire opposés, mais un processus de discussion médiatisé par la recherche d'informations pertinentes (enquêtes pragmatiques) et par l'usage d'instruments (outils d'analyse, interven- 
tion d'un tiers externe) peut aboutir à une mise en cohérence partielle des schèmes, avec d'autant plus de chance de succès qu'aucun acteur n'est en position d'accusé ni d'accusateur. Un processus de tertiarisation permet de remplir cette condition, car la nécessité du changement provient alors d'un acteur extérieur à l'organisation (le client), fortement légitime, et le récit met en scène les problèmes qu'il rencontre et la production de solutions à ces problèmes.

- La transformation par les acteurs de leurs schèmes d'interprétation dans l'interaction avec les autres acteurs porteurs d'autres schèmes est d'autant plus profonde qu'ils sont les auteurs du nouveau récit. L'éventuel intervenant externe, consultant ou chercheur, n'est qu'un facilitateur (il joue un rôle instrumental).

Nous présenterons tour à tour deux exemples pris dans des secteurs de nature très différente: l'industrie informatique et la filière de la viande porcine. Dans ces deux exemples, les auteurs du présent article ont été des acteurs directs, en tant que cadre supérieur d'un groupe informatique dans un cas, en tant qu'expert et conseil dans l'autre cas. Les deux cas sont ainsi issus d'une démarche de rechercheaction.

\section{II. - UN PREMIER EXEMPLE : L'INDUSTRIE INFORMATIQUE}

\section{La tertiarisation de l'industrie informatique}

À la fin des années 1980, l'industrie informatique se tertiarise. Jusqu'alors, avec les générations d'ordinateurs « mainframe » ou « mini-ordinateurs » (IBM 360 puis AS 400, Bull DPS 7), les entreprises du secteur (IBM, Digital, Hewlett Packard, Fujitsu, Bull, etc.) apparaissaient comme des industriels manufacturiers au sens le plus classique du terme, même s'ils mobilisaient des technologies de pointe: ils concevaient et vendaient des gros ordinateurs qui s'identifiaient à des « moteurs informationnels », supports de mémorisation et de calcul, biens d'équipement lourds, « machines-outils » au sens littéral du terme (des machines qui servaient d'outils à des utilisateurs professionnels), fortement personnalisés (des machines du même modèle destinées à des clients différents étaient très différentes), à forte valeur ajoutée industrielle, d'utilisation techniquement complexe mais pauvre fonctionnellement (les utilisateurs devaient être des informaticiens, la machine servait à automatiser des traitements d'information massifs et répétitifs, la complexité de l'usage était repoussée vers l'utilisateur). L'importance industrielle de l'ingénierie spécifique et des processus de production (notamment des tests fonctionnels) faisait incontestablement des ordinateurs des biens manufacturés très élaborés.

Le progrès technique permit, au fil des années 1980 et 1990 , de fortement développer les capacités de mémorisation et de traitement de l'information et de simplifier considérablement les technologies de conception (standardisation des composants, des logiciels d'exploitation), de production et d'utilisation de ces machines. Le produit s'est ainsi transformé de bien d'équipement lourd en bien intermédiaire (destiné à des utilisateurs grand public tout autant qu'à des utilisateurs professionnels). Les capacités de mémorisation et de traitement de l'information se sont banalisées, devenant des 
« commodities », ressources primaires de faible valeur. Les puissances immenses désormais accessibles à un coût modeste rendent possibles la reconception profonde de la base informationnelle des organisations, et, en fait, à travers l'information, la reconception des organisations ellesmêmes, avec des effets structurants, comme le montre le marché des logiciels de gestion intégrés (ERP).

L'érosion de la valeur ajoutée industrielle est accélérée par la standardisation des PC (quasi-monopole de Windows). Par contre, le besoin d'ingénierie des systèmes d'information et d'organisation se développe rapidement, entraînant une croissance forte du marché des services. L'industrie informatique se reconfigure autour de la notion de solution, combinaison de produits matériels et logiciels et de services à forte spécificité. Aujourd'hui, IBM, l'un des seuls « grands » de l'informatique traditionnelle qui aient survécu, est devenu une entreprise de conseil et de service.

\section{La phase de transition et ses difficultés}

La phase de transition de l'informatique « lourde » vers l'informatique « immatérielle » a posé de redoutables problèmes aux entreprises piégées dans leur culture technologique (le métier informatique est alors identifié à la capacité de développer des systèmes matériels et un système d'exploitation performants) et dans leur culture manufacturière. Ce piège constitue une menace mortelle. Peu de groupes ont négocié la mutation avec succès (en dehors d'IBM, fortement chahuté, et Hewlett Packard, presque tous les constructeurs: Digital, Unisys, Bull, Control Data, ont sombré dans la tempête). Mentionnons quelques exemples des changements de perspective requis (Lorino, 2000) :

- l'activité de service, vue traditionnellement comme un complément de la vente de matériel, devient l'activité principale et doit conquérir son autonomie stratégique de pouvoir et de moyens au sein de l'organisation,

- démarche commerciale: liberté de vendre du service sur les matériels des concurrents, mise en place d'un réseau commercial dédié au service,

- gestion: déconsolidation du service et du matériel dans le reporting,

- compétences: réorientation des compétences vers le diagnostic et la conception de systèmes,

- gestion industrielle: la faiblesse de la valeur ajoutée industrielle comparée à la valeur élevée des matériels achetés et manipulés apparente de plus en plus le secteur à une activité logistique dans laquelle les considérations de flux l'emportent largement sur la transformation technique de la matière; cela implique que l'on raffine la gestion des stocks et des flux et que l'on simplifie radicalement la gestion de la production elle-même, en imitant les modes de gestion de la grande distribution...

Ces transformations heurtent bien sûr les intérêts, les cultures et les métiers établis.

\section{3. Échecs et succès}

Toutes les entreprises du secteur, y compris les « survivants » (IBM et HP), ont connu une phase de crise. Surmonter cette crise n'a été possible que par une reconception radicale de l'organisation: changement de dirigeants, transformation massive des profils de métiers, modifications de structures. Il s'agissait de redessiner les chaînes de valeur, ce qui devait s'accompagner d'une 
remise en cause profonde des référentiels de sens: produit-on de la richesse en transformant la matière pour créer des objets quasiment magiques, ou en structurant et résolvant les problèmes des clients? Les systèmes de significations s'en trouvent profondément modifiés, par exemple:

- le rendement et les coûts de production n'ont qu'une importance subalterne, l'essentiel résidant dans l'agilité du flux,

- le client n'est pas un spécialiste de l'informatique, mais son problème étant le noyau de la future affaire, l'écouter est fondamental,

- les machines sont, à peu de choses près, interchangeables, l'avantage concurrentiel ne peut résider que dans les compétences que l'entreprise déploie dans le service.

La plupart des groupes du secteur tinrent alors des discours stratégiques similaires sur la tertiarisation de l'informatique, mais peu s'avérèrent capables de traduire ces nouveaux référentiels de sens, formulés au plus haut niveau par un petit nombre de dirigeants, dans des systèmes de signes cohérents, des nouveaux langages et donc des modes d'action réellement transformés. L'interprétation et la mise en actes du discours stratégique dans l'espace des métiers et des opérations firent défaut. Le discours stratégique était condamné à demeurer incantatoire, car les dirigeants ne trouvaient pas les voies et moyens d'une reconstruction des significations et des schèmes d'interprétation, dans les profondeurs de l'entreprise. De plus, dans les grands groupes, l'organisation bureaucratique fait souvent écran aux clients: la mutation du marché est analysée de manière pertinente au plan théorique, mais elle ne s'impose pas physiquement et affectivement, « existentielle- ment », aux acteurs, comme impératif de bouleverser les registres de signification.

\section{Le rôle des récits}

Pour illustrer l'importance de l'enquête comme processus narratif collectif, nous nous intéresserons au processus de vente. Ce processus implique divers acteurs: le vendeur, l'ingénieur commercial qui l'assiste dans la vente sur les aspects les plus techniques et assure l'installation de la machine vendue, l'ingénieur technico-commercial qui résout les problèmes techniques plus complexes, notamment liés à la configuration spécifique au client et aux évolutions et mises à jour des versions, l'ingénieur de service spécialisé dans le type d'application mis en œuvre par le client. Traditionnellement, le vendeur se perçoit comme acteur-clé d'un récit où ses exploits consistent à vendre un maximum de machines «magiques » (les ordinateurs ont alors une aura particulière). Le dernier modèle de machine et le plus beau, celui qu'il faut parvenir à faire désirer par les clients. L'ingénieur commercial intervient dans le cadre d'un récit où il détient des connaissances sur les machines vendues qui permettent de convaincre le client de sa compétence et ainsi de le rassurer sur l'adéquation de la machine à sa situation technique. L'ingénieur technico-commercial se voit comme le sorcier auquel ses collègues et le client feront appel lorsqu'ils se heurtent à des questions plus énigmatiques car plus complexes. Il orchestre l'intégration et les évolutions. L'ingénieur de service doit, si besoin est, apporter le soutien d'une solution logicielle, y compris de solutions logicielles très spécifiques et pointues désirées par le client et fournies par quelque PME 
inconnue, pour débloquer la vente de la machine.

Mais soudain, dans les situations nouvellement vécues, le récit du client devient déstabilisateur. «La solution informatique que vous m'avez fournie ne permet pas de faire fonctionner correctement la comptabilité de mon entreprise. Je ne veux pas savoir si c'est un problème de puissance de traitement installée, de fiabilité technique du matériel, de qualité ou de pertinence du logiciel, ce n'est pas mon problème. Qui plus est, le logiciel de comptabilité qui doit tourner sur cette machine n'est plus maintenu car la PME qui l'a développé a fait faillite. Donc l'ordinateur que vous m'avez vendu ne me sert à rien. »

En présence de ce discours du client, les récits antérieurs des acteurs du processus éclatent. Chacun tente de le reconstruire à sa façon. Le vendeur se lamente: « je vais perdre mon client parce que le support service n'a pas fait son travail », l'ingénieur commercial: " les machines ont été parfaitement installées mais le vendeur et l'ingénieur de service n'ont pas bien choisi le fournisseur de logiciel », l'ingénieur de service: « les besoins de ce client n'ont pas été correctement identifiés par le vendeur et l'ingénieur commercial et la puissance installée ne répond pas aux besoins »... Dans un univers de vente de matériel éventuellement étayée par la fourniture accessoire de logiciels et de services, les récits des acteurs deviennent inconciliables et sont mis en échec par la réfutation obstinée par le client. La reconstruction de l'organisation et des logiques d'action doit passer par l'élaboration collective d'un nouveau récit intégrateur ( nous sommes les acteurs d'une chaîne de services qui doit faire feu de tout bois - toute machine, tout logiciel, toute étude technique ou applicative - pour répondre à un besoin bien identifié du client »). Ce récit se traduira par des « mises en intrigue » propres à chaque acteur profondément transformées, de nouvelles priorités, de nouvelles modalités de coordination et donc de nouveaux rapports de pouvoir au sein du processus. Peu d'organisations se sont historiquement révélées capables de franchir cette étape.

\section{III. - UN SECOND EXEMPLE : LA FILIÈRE PORCINE}

Dans ce deuxième exemple, nous décrivons d'abord brièvement le processus de tertiarisation dans les filières agroalimentaires, notamment la filière porcine. Puis, nous explicitons comment une approche pragmatique conduite par les éleveurs et les salariés leur a permis de piloter le processus de tertiarisation de leur filière. Enfin, une analyse sémiotique des discours montre le rôle du récit dans les changements opérés.

\section{La tertiarisation de l'agriculture}

Comme dans l'industrie informatique, l'agriculture est engagée, au tournant des années 1990, dans une mutation qui est aussi importante que celle qu'elle a connue avec l'« industrialisation » à partir des années 1960. Cette mutation repose sur une tertiarisation de l'agriculture (Nefussi, 1999; Nefussi et Priolon, 1999.

Dans les années 1960, l'agriculture s'industrialise en remettant en cause les « savoirs paysans »par des connaissances scientifiques et techniques, en substituant du capital à du travail et en augmentant les intrants (produits phytosanitaires, alimentation animale, etc.). Cette industrialisation a permis d'abaisser les coûts de production par 
l'augmentation de la productivité. Cette mutation n'a rien d'original, l'industrie a accompli une transformation identique. Il y a « industrialisation » dans la mesure où l'agriculture incorpore des concepts et des méthodes qui sont les schèmes d'interprétation courants de l'industrie à l'époque. Cela implique, par exemple, l'introduction de la comptabilité analytique pour calculer des coûts ou de la comptabilité générale pour pouvoir emprunter. Enfin, avec l'industrialisation, l'agriculture entre dans une logique de marché avec une concurrence entre producteurs basée sur les coûts.

Aujourd'hui, pour répondre aux nouvelles attentes des consommateurs-citoyens en matière d'alimentation et d'environnement, l'agriculture doit s'engager dans une mutation de même ampleur: elle doit incorporer de plus en plus « des concepts et des méthodes » qui proviennent du secteur tertiaire. Cette tertiarisation se manifeste dans trois domaines :
- l'agriculteur devient un prestataire de services marchands: tourisme vert (restauration, hébergement, etc.), travaux agricoles en sous-traitance ;

- l'agriculteur devient un prestataire de services non marchands: paysage, occupation du territoire, préservation de l'environnement, etc ;

- la production agricole est « tertiarisée » avec l'arrivée du «produit-service » agricole, instrument matériel d'une «solution agricole ».

Pour illustrer ce processus de tertiarisation de la production agricole, on peut s'appuyer sur les changements que connaissent les entreprises de la filière des viandes: éleveurs, groupement de producteurs, abattoirs, ateliers de découpe.

\section{Les enjeux stratégiques de la} tertiarisation de la filière des viandes

À la suite du développement du libre-service dans la distribution de détail de

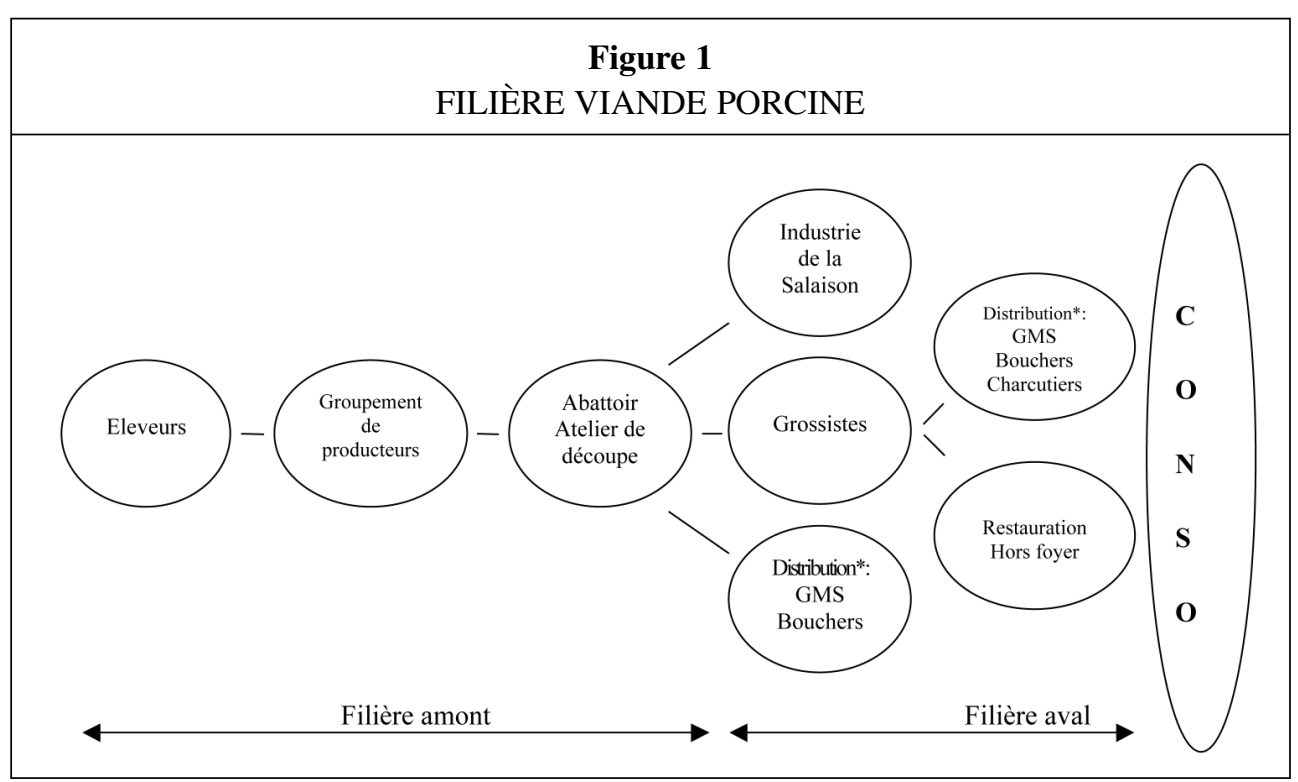

Note: * La distribution peut être un client direct ou indirect des abattoirs et ateliers de découpe. 
viandes et de l'industrialisation de la filière (années 1970-1990), les fournisseurs de la grande distribution ${ }^{1}$ ont perdu la capacité de prescription de la viande: la conception du produit, la définition de ses attributs sont définis par la distribution à travers ses cahiers des charges. Ainsi, sur le marché de la viande de porc, le produit est banalisé et les stratégies de coûts dominent l'univers concurrentiel. Mais, l'évolution des attentes des consommateurs et les changements d'ordre réglementaire consécutifs à la crise de l'ESB remettent en cause les équilibres entre les entreprises de la filière des viandes. La prise en compte de ces problèmes crée de nouvelles possibilités de prescription: une offre de solutions agricoles. Comme toute offre de services, la solution agricole n'existe pas positivement, elle est à construire avec le client.

Ainsi, au tournant des années 2000, les entreprises amont des filières agro-alimentaires doivent engager un profond changement pour vendre des « solutions agricoles » plutôt que des produits agricoles. Les principaux éléments de cette évolution sont:

- une coopérativité fondée sur une connaissance partagée des attentes des clients directs et indirects par les acteurs de la chaîne de valeur (éleveurs, salariés des différentes entreprises de la filière),

- une reconfiguration des processus de production au sein des filières amont par rapport à ces attentes qui donnent un sens différent à la production: évolution des compétences, des organisations, des coordinations,

- une nouvelle instrumentation des actions conjointes au sein de la filière: systèmes d'informations collaboratifs, langages communs (codifications communes, critères d'évaluations et de contrôle, etc.), organisation des interfaces, etc.

La tertiarisation de l'activité pour les éleveurs comme pour les salariés des entreprises n'implique pas seulement la mise en œuvre de nouvelles méthodologies, mais un changement complet des schèmes d'interprétation de leurs activités.

\section{Le cas d'un groupement de producteurs de porcs}

Le Groupe APS-SPM est composé d'un groupement de producteurs de porcs et d'une société d'abattage et de découpe. Le Groupe est engagé dans de nombreuses démarches qualité :

- traçabilité complète du processus de production (de l'éleveur à la caissette),

- certification de conformité de produit: «ARCA » et « Saveur Gourmande »,

- appellation « Montagne »,

- qualification des élevages: 80-90\% des élevages.

Le positionnement commercial du Groupe vise à la fois des segments de marché sous signes officiels de qualité (IGP « Jambon de Bayonne » ou AOC «Lacaune »), et la grande distribution: Intermarché, Casino, Leclerc, Système U, etc.

La multiplication des cahiers des charges, notamment avec les signes de qualité, a été la première réponse à un positionnement de plus en plus concurrentiel. Cette stratégie a reposé sur la croyance que la valeur du produit est d'autant élevée que le cahier des charges est complexe. La crise porcine et la chute des marges ont entamé cette croyance et justifié une réflexion critique, fondée par 
le processus de tertiarisation. Cette demande de réflexivité s'est traduite par la commande d'un audit «produit-service $»^{2}$ par la direction du groupe, avec les objectifs suivants :

- augmenter la valeur ajoutée du Groupe et le revenu des éleveurs,

- rapprocher les éleveurs de la réalité du marché de la viande,

- gagner en efficacité économique,

- simplifier le processus de production (réduction du nombre de cahiers des charges),

- définir le socle des marques propres et l'implication commerciale chez les clients,

- valider les orientations commerciales (signes de la qualité et marques).

Analysée par référence à la tertiarisation, cette combinaison d'objectifs conduit à un bouleversement complet du «métier » du Groupe et de la chaîne de valeur. C'est une remise en cause radicale des croyances et de la culture professionnelle des éleveurs et des salariés.

\section{Enquêtes pragmatiques et organisation de la réflexivité dans les actions conjointes}

L'audit « produits-services » doit permettre aux acteurs de la chaîne de valeur, ici les éleveurs et les salariés du Groupe, d'élaborer eux-mêmes un nouveau schème d'interprétation de leur activité et de redéfinir les actions collectives conjointes. En pratique, il conduit à l'élaboration par les salariés et les éleveurs d'un nouveau récit intégrateur qui se concrétise par un projet et un plan d'actions.
Le récit initial repose sur une croyance: « la viande de porc est un bien banal, son usage est évident pour chacun, son utilisation ne constitue pas un domaine d'interrogation qui puisse être une source d'innovation et de différenciation; le coût de production est le seul facteur clés de succès. Seuls les signes de qualité permettent de desserrer cette contrainte ». L'audit remet d'abord en cause le schème initial par la formulation d'un « récit diagnostic ». Dans un deuxième temps, l'audit favorise une nouvelle intelligibilité des actions conjointes par l'élaboration d'un « récit utopique » qui se concrétise sous la forme d'un plan d'actions.

Dans une logique de production de solutions, la connaissance des attentes des clients directs et indirects prend une importance considérable puisqu'elle soutient tout le processus de prescription et de réorganisation des processus de production. Or cette connaissance est difficile à acquérir par la filière amont car la viande acquiert de la valeur par la capacité qu'ont les acteurs de l'aval de l'utiliser. Il faut donc connaître les conditions effectives de consommation et d'utilisation chez les différents clients pour élaborer des solutions pertinentes. La division économique du travail est un obstacle objectif à cette compréhension partagée.

L'audit prend la forme d'un programme de travail collaboratif qui associe des éleveurs et des salariés du Groupe APS-SPM. Le programme comporte trois composantes: - Une enquête pragmatique sur les attentes des clients: il ne s'agit ni de contrôler ou d'évaluer la situation initiale, ni d'élaborer une quelconque vérité. Les enquêtes ne

2. La direction du groupe a souhaité l'intervention d'un consultant pour réaliser un audit «produit-service » qui articule une explicitation de la tertiarisation et une recherche de coopérativité renforcée au sein de la filière par un accompagnement de la réflexivité étendue aux fournisseurs agricoles. 
visent que la construction du sens de l'activité («A quoi sert le produit? ») et le plan d'actions.

- Des recommandations pour agir sur les processus internes et sur les processus collaboratifs au sein du Groupe. Ces processus sont interprétés par les acteurs comme actions collectives conjointes.

- La communication des résultats à tous les acteurs de la chaîne de valeur du groupe : le nouveau récit prend la forme d'un projet collectif et d'un plan d'actions qui doivent être partagés au-delà des participants à l'audit.

Sur un plan méthodologique, un " dispositif projet » est mis en place. Il comporte un chef de projet (le directeur du groupe), un comité de pilotage composé d'éleveurs, de cadres et de salariés, et des groupes de travail formés de personnes appartenant aux différentes fonctions de l'entreprise. Ces groupes de travail réalisent les analyses, ils sont responsables des recommandations qui les concernent directement. Deux types de travaux sont réalisés :

- une enquête de terrain auprès des clients pour comprendre la nature de leurs attentes, - une analyse critique des actions conjointes au sein de la chaîne de valeur pour élaborer les solutions attendues par les clients.

Dans ce dispositif, le rôle du consultant est triple:

- Présenter le processus global de tertiarisation de la filière des viandes pour engager la déconstruction du schème d'interprétation initial de l'activité courante. Il en résulte une prise de conscience collective de l'ignorance des conditions réelles d'utilisation des animaux par les éleveurs et de la viande par les éleveurs et les salariés.
- Organiser la production de connaissances « nouvelles » par les groupes de travail sur les problèmes à résoudre chez les différents types de clients :

- organiser une enquête effectuée par 20 binômes (un éleveur et un salarié) auprès de 50 clients à l'aide de questionnaires semi-directifs,

- effectuer les traitements statistiques des questionnaires,

- rédiger la synthèse qui est à la fois le récit du mode de production des résultats de l'enquête et la nouvelle représentation des attentes des clients.

- Organiser la reconfiguration des processus collaboratifs par les groupes de travail: - définir la méthodologie d'analyse des processus et les types de résultats attendus (réorganisation, acquisition de compétences, modification du système d'information, investissements, etc.),

- composer les groupes de travail,

- rédiger le plan d'actions à partir des conclusions intermédiaires.

Dans ce processus réflexif sur la coopérativité dans les actions conjointes, le consultant n'est pas un expert qui sait ce que le groupe doit faire, il n'est pas non plus un clinicien chargé de mettre en évidence les causes de dysfonctionnement. Son rôle se limite à l'organisation méthodologique d'une approche pragmatique et réflexive. Si la reconfiguration des processus dans le cadre d'une conduite participative du changement et le plan de communication n'appellent pas de remarques particulières, parce qu'ils reposent sur des outils bien connus de conduite du changement, il faut noter que la phase initiale est en fait fondée sur l'élaboration collective d'un nouveau récit. 
Les acteurs qui participent à l'enquête auprès des clients sont: les éleveurs, les opérateurs de la chaîne d'abattage, les préparateurs de commandes, un informaticien, des responsables de la logistique amont et aval, des qualiticiens, des commerciaux, des administratifs. Contrairement à la routine, ces personnes sont mises dans une situation de production de connaissances (élaboration du questionnaire et enquête sur le terrain) qui remet en cause des habitudes d'interprétation de l'activité tenues pour évidentes: la complexification du cahier des charges au stade de l'élevage n'intéresse personne; l'origine Aveyron est importante, contrairement à l'appellation « Montagne »; les clients n'utilisent pas la viande de la même manière; des détails ont une importance considérable dans le processus de transformation ou de commercialisation; les heures et les jours de livraison peuvent être optimisés pour chaque client; le prix n'est pas le premier critère pris en compte pour plus de la moitié des clients... Un nouveau schéma narratif peut être imaginé: le client a des problèmes que notre produit peut aider à résoudre, ce peut être la base d'un nouveau positionnement concurrentiel.

Dans la déconstruction du récit ancien et dans l'élaboration du nouveau récit, les paroles échangées et le langage jouent un rôle instrumental essentiel. L'enquête, sa préparation et son déroulement sont vécus par les participants comme une expérience personnelle nouvelle qui valide ou remet en cause les opinions de chacun. La synthèse statistique permet de dépasser la perception individuelle de la réalité. Le débat autour des résultats permet une appropriation beaucoup plus profonde que les résultats d'une enquête réalisée par des professionnels extérieurs à l'entreprise. En effet, les dialogues interpersonnels conduisent à reconstruire les systèmes de signification. Par exemple, dans la préparation de l'enquête, il y a d'abord la prise de conscience que les mêmes termes ne désignent pas les mêmes choses pour les différents services. Une sémantique commune se constitue dans les dialogues. Ensuite, le caractère collectif de la production de connaissances permet une appropriation par les acteurs des nouvelles contraintes qui sont issues de la demande des clients et donnent un sens à l'activité quotidienne.

\section{CONCLUSION}

En situation de tertiarisation, le nouveau récit intégrateur construit à travers l'enquête collective « relit » le passé sans le renier mais en le réinterprétant dans une perspective de changement. Il se cristallise dans de nouveaux types d'instruments, qui consolident les nouveaux schèmes interprétatifs et prolongent le récit fondateur dans la création de nouvelles habitudes d'action. La remise en cause touche les fondements même de la révolution industrielle, puisqu'il ne s'agit plus seulement de produire de manière moins coûteuse, mais de manière plus « pertinente ». Dans les économies tertiarisées, la compétitivité d'une entreprise repose sur sa capacité à se saisir des problèmes pour construire des solutions. La compétitivité de l'offre réside dans sa pertinence, issue directement des compétences collectives mobilisées et d'une construction de connaissances à jet plus ou moins continu par les acteurs humains de la chaîne de valeur. La solution est toujours une production entre humains. Les outils facilitent l'action interpersonnelle mais ne peuvent pas se substituer à elle. 
Cette mutation, d'un point de vue pratique, recouvre deux mouvements complémentaires :

- la mise au point de nouvelles pratiques managériales, notamment dans l'organisation d'enquêtes réflexives sur les modes d'action collectifs, la « mise en processus » et en récit des actions séparées contribuant à la création de valeur,

- une nouvelle approche de l'instrumentation de gestion, comme support de sens, dont la conception doit explicitement prendre en compte les schèmes d'utilisation futurs visés, plutôt que de tenter d'atteindre une « vérité scientifique » dans la réplication et la mesure du monde réel.

Pour rendre compte de ces mutations, les approches pragmatiques et sémiotiques permettent d'éclairer le changement organisationnel lié à la tertiarisation en surmontant les limites propres à la séparation traditionnelle entre échange marchand et processus d'action organisé.

\section{Bibliographie}

Allaire P., "The CEO as Organizational Architect: An Interview with Xerox's Paul Allaire", Harvard Business Review, vol. 70, $\mathrm{n}^{\circ}$ 5, septembre 1992, p. 106-121.

Argyris Ch., Schön D., Organizational Learning. A Theory of Action Perspective, AddisonWesley, New York, 1978.

Charaudeau P., Maingueneau D., Dictionnaire d'analyse du discours, Seuil, Paris 2002.

Clot Y., « De Vygotski à Leontiev, via Bakhtine », Avec Vygotski, ouvrage collectif sous la direction de Clot Y., La Dispute, Paris, 1999/2002.

Clot Y., Faïta D., « Genre et style en analyse du travail », Travailler, n 3, 2000.

De Bandt J., Gadrey J., Relations de services, marchés de services, CNRS Édition, coll. « Recherche et Entreprise », Paris, 1994.

De Bandt J., Les services : productivité et prix, Economica, Paris, 1991.

Dewey J., Logique. La théorie de l'enquête, Presses Universitaires de France, Paris, 1993.

Gadrey J., Delaunay J.-C., Les enjeux de la société de service, Presse de la Fondation nationale des sciences politiques, Paris, 1987.

Gadrey J., Zarifian Ph., L'émergence d'un modèle du service, éditions Liaisons, décembre 2001.

Levitt Th., "Marketing Intangible Products and Product Intangibles", Harvard Business Review, vol. 59, n³, mai-juin 1981, p. 94-102.

Lorino Ph., «L'apprentissage organisationnel bloqué: le cas de Bull »Enquêtes de gestion. À la recherche du signe dans l'entreprise, ouvrage collectif sous la direction de $\mathrm{Ph}$. Lorino, L'Harmattan, Paris, 2000.

Lorino Ph., Demeestère R., Genestet V., « Penser la gestion des compétences », L'Expansion Management Review, ${ }^{\circ}$ 110, septembre 2003, p. 72-78.

Nefussi J., Priolon J., "Coordination Without Vertical Integration: the Case of the French Food System", Vertical Relationships and Coordination in the Food System, ouvrage collectif sous la direction de G. Galizzi et L. Venturini, Physical Verlag, Heidelberg, 1999. 
Nefussi J., « Filières agroalimentaires : filières de produits ou filières de services ? », Demeter 2000, Armand Colin, Paris, 1999.

Nonaka I., Takeuchi H., The Knowledge-Creating Company, Oxford University Press, Oxford, 1995.

Peirce Ch. S., Écrits sur le signe, Le Seuil, Paris, 1978.

Rabardel P., «Le langage comme instrument? Éléments pour une théorie instrumentale étendue. », Avec Vygotski, ouvrage collectif sous la direction d'Yves Clot, La Dispute, Paris, $1999 / 2002$.

Ricœur P., Temps et récit, tome I: L'intrigue et le récit philosophique, Le Seuil, Paris 1984.

Vernant D., Du discours à l'action. Études pragmatiques, Presses Universitaires de France, Paris, 1997.

Vygotski L., Pensée et langage, La Dispute, Paris, 1997.

Weick K., Making Sense of the Organization, Blackwell, Oxford (UK) et Malden (MA, USA), 2001. 